\title{
Molecular characterization and protective efficacy of the microneme 2 protein from Eimeria tenella
}

\author{
Ming Yan ${ }^{1,2}$, Xiaoxia Cui ${ }^{1,3}$, Qiping Zhao ${ }^{1}$, Shunhai Zhu ${ }^{1}$, Bing Huang ${ }^{1}$, Lu Wang ${ }^{1}$, Huanzhi Zhao ${ }^{1}$, Guiling Liu ${ }^{1}$, \\ Zhihang $\mathrm{Li}^{1,2}$, Hongyu Han ${ }^{1}$, and Hui Dong ${ }^{1, *}$ \\ ${ }^{1}$ Key Laboratory of Animal Parasitology of Ministry of Agriculture, Shanghai Veterinary Research Institute, CAAS, \\ Shanghai 200241, PR China \\ 2 College of Life and Environment Sciences, Shanghai Normal University, Shanghai 200234, PR China \\ ${ }^{3}$ Qingdao Yebio Biological Engineering Co., Ltd, Qingdao 266114, PR China
}

Received 21 August 2018, Accepted 12 November 2018, Published online 26 November 2018

\begin{abstract}
Microneme proteins play an important role in the adherence of apicomplexan parasites to host cells during the invasion process. In this study, the microneme 2 protein from the protozoan parasite Eimeria tenella (EtMIC2) was cloned, characterized, and its protective efficacy as a DNA vaccine investigated. The EtMIC2 gene, which codes for a $35.07 \mathrm{kDa}$ protein in E. tenella sporulated oocysts, was cloned and recombinant EtMIC2 protein (rEtMIC2) was produced in an Escherichia coli expression system. Immunostaining with an anti-rEtMIC2 antibody showed that the EtMIC2 protein mainly localized in the anterior region and membrane of sporozoites, in the cytoplasm of first- and second-generation merozoites, and was strongly expressed during first-stage schizogony. In addition, incubation with specific antibodies against EtMIC2 was found to efficiently reduce the ability of $E$. tenella sporozoites to invade host cells. Furthermore, animal-challenge experiments demonstrated that immunization with pcDNA3.1(+)-EtMIC2 significantly increased average body weight gain, while decreasing the mean lesion score and oocyst output in chickens. Taken together, these results suggest that EtMIC2 plays an important role in parasite cell invasion and may be a viable candidate for the development of new vaccines against $E$. tenella infection in chickens.
\end{abstract}

Key words: Coccidiosis, Eimeria, microneme-2, EtMIC2, invasion, immunization.

Résumé - Caractérisation moléculaire et efficacité protectrice de la protéine de micronème 2 d'Eimeria tenella. Les protéines des micronèmes jouent un rôle important dans l'adhésion des parasites Apicomplexa aux cellules hôtes au cours du processus d'invasion. Dans cette étude, la protéine de micronème 2 du protozoaire parasite Eimeria tenella (EtMIC2) a été clonée, caractérisée et son efficacité protectrice en tant que vaccin à ADN a été étudiée. Le gène EtMIC2, qui code pour une protéine de $35.07 \mathrm{kDa}$ dans des oocystes sporulés d'E. tenella, a été cloné et la protéine EtMIC2 recombinante (rEtMIC2) a été produite dans un système d'expression d'Escherichia coli. Une immuno-coloration avec un anticorps anti-rEtMIC2 a montré que la protéine EtMIC2 se localisait principalement dans la région antérieure et la membrane des sporozoïtes, dans le cytoplasme des mérozoïtes de première et de deuxième génération, et était fortement exprimée au cours du premier stade de schizogonie. De plus, une incubation avec des anticorps spécifiques contre EtMIC2 s'est avérée efficace pour réduire la capacité des sporozoïtes d'E. tenella à envahir les cellules hôtes. En outre, des expériences d'infestation ont montré que l'immunisation avec pcDNA3.1(+)-EtMIC2 augmentait de manière significative le gain de poids corporel moyen, tout en diminuant le score de lésion moyen et l'excrétion d'oocystes des poulets. Pris dans leur ensemble, ces résultats suggèrent qu'EtMIC2 joue un rôle important dans l'invasion de cellules parasitaires et pourrait constituer un candidat viable pour la mise au point de nouveaux vaccins contre l'infection à E. tenella chez les poulets.

\section{Introduction}

Avian coccidiosis, a protozoan parasitic disease caused by the intracellular apicomplexan parasite, Eimeria spp., leads to heavy economic losses in the poultry industry worldwide [5]. It causes an estimated loss of more than $\$ 3$ billion USD per

\footnotetext{
*Corresponding author: donghui@shvri.ac.cn
}

annum due to production losses and veterinary prophylactic measures [1,33]. Poultry farmers mainly rely on the use of "coccidiostat" in the feed to treat and/or prevent Eimeria infection. However, rigorous use of anticoccidial drugs has led to the development of drug-resistant Eimeria strains $[4,23]$. The second most effective way to prevent coccidiosis is the use of live anticoccidial vaccines; however, until recently the use of these vaccines has been limited to broiler and layer 
breeders only due to the limited production, chances of virulence reversibility, and high cost $[1,31]$. Therefore, there has been an increased effort to develop new control strategies for Eimeria infection that target multiple stages of the parasitic invasion process. One of these approaches is to block the invasion of Eimeria into intestinal epithelial cells to prevent coccidiosis.

Eimeria spp. belong to the apicomplexan parasites, possessing a characteristic apical complex consisting of micronemes, rhoptries, and structural elements such as the conoid, polar ring, and subpellicular microtubules [27]. Micronemes are small membrane-bounded organelles located immediately beneath the cell membrane, near the anterior end of the apical complex, and release numerous soluble and transmembrane proteins [41]. Previous studies have shown that the proteins secreted by micronemes are involved in multiple interactions between the parasite and the host cell, specifically in relation to motility, attachment, recognition, and penetration, and thus play a crucial role in the invasion process of apicomplexan parasites $[2,3,11$, $22,25,35,39]$. The $E$. tenella microneme-2 gene (EtMIC2) was first identified by Tomley et al. [39] and since then several studies have suggested that EtMIC2 has good immunogenicity and may be a good vaccine candidate [6, 29, 32, 36, 45, 47]. In this study, EtMIC2 was cloned, characterized, and its protective efficacy as a DNA vaccine investigated.

\section{Materials and methods \\ Ethics Statement}

Coccidia-free chickens and rabbits were used in this study. The protocol was approved by the Animal Care and Use committee of the Shanghai Veterinary Research Institute, Chinese Academy of Agricultural Sciences. The animals were provided with water and food ad libitum. At the end of the experiments, the animals were euthanized in strict accordance with the international standards for animal welfare.

\section{Experimental chickens, parasites, and cells}

One-day-old yellow feathered broilers were kept in wire cages under coccidia-free conditions and provided with coccidiostat-free feed and water ad libitum. Periodic examination of Eimeria infection of the chickens was done by microscopic examination of feces. The chickens were moved to an animal containment facility prior to the challenge with virulent oocysts.

Eimeria tenella was isolated from Shanghai [10] and stored in the Key Laboratory of Animal Parasitology at the Ministry of Agriculture, Shanghai Veterinary Research Institute of the Chinese Academy of Agricultural Sciences. These parasites were maintained and propagated in two-week-old coccidia-free chickens, as previously described [40]. Sporulated oocysts were obtained and purified using standard procedures [8]. Sporozoites were obtained from cleaned sporulated oocysts with in vitro excystation, and were purified using chromatography over columns packed with nylon wool and DE-52 cellulose [9]. Second generation merozoites were collected and purified from the cecal mucosa at $112 \mathrm{~h}$ post-inoculation from chickens inoculated with $1 \times 10^{5}$ sporulated oocysts [34].

The chicken embryo fibroblast cell line, DF-1, was maintained and cultured in Dulbecco's modified Eagle's medium (DMEM) (Invitrogen, Carlsbad, CA, USA) supplemented with $10 \%$ fetal bovine serum (FBS) at $37^{\circ} \mathrm{C}, 5 \% \mathrm{CO}_{2}$ [13].

\section{Cloning of the EtMIC2 gene}

A total of $1.0 \times 10^{7}$ sporulated oocysts of E. tenella were ground using a pre-chilled mortar and pestle. Total RNA was isolated from sporulated oocysts using TRIzol (Invitrogen), according to the manufacturer's protocol. To avoid DNA contamination, the extracted RNA preparations were treated additionally with RNase-free DNase I (Takara, Dalian, China) for $30 \mathrm{~min}$ at $37^{\circ} \mathrm{C}$. DNase I was then inactivated by heating at $75^{\circ} \mathrm{C}$ for $10 \mathrm{~min}$. RNA was quantified with UV spectrophotometry (Eppendorf, Hamburg, Germany), and its integrity verified with electrophoresis using a $1 \%$ agarose denaturing formaldehyde-ethidium bromide (EtBr) gel. Complementary DNA (cDNA) was synthesized from the total RNA using an M-MLV Reverse Transcriptase kit (Invitrogen).

The complete coding region of the EtMIC2 gene was amplified using primers designed with Primer Premier version 5.0 software according to the mRNA sequence published in GenBank (accession number: AF111839.1). The primer sequences were as follows: forward primer, 5'-CGGGATCCGTTGCATTGCATAACCTCAT- ${ }^{\prime}$; reverse primer, 5'-CCCTAAGCTCGTCACTCTGCTTGAACCT-3', containing BamHI and HindIII restriction sites (bold), respectively. Amplification was performed by an initial reaction at $95{ }^{\circ} \mathrm{C}(5 \mathrm{~min})$ followed by 30 cycles of $95{ }^{\circ} \mathrm{C}(30 \mathrm{~s}), 48^{\circ} \mathrm{C}(30 \mathrm{~s}), 72^{\circ} \mathrm{C}$ (1 min), and a final extension at $72{ }^{\circ} \mathrm{C}(10 \mathrm{~min})$. After amplification, gel electrophoresis was carried out and targeted bands were selected, purified, and ligated to the pGEM-T easy cloning vector (Promega, Madison, USA), according to the manufacturer's instructions. These vectors were transformed to TOP10 Escherichia coli. Positive colonies were picked from the plates and checked using enzymatic digestion using the previously inserted restriction sites, followed by sequence confirmation by Invitrogen Bio-tech (Shanghai Huajin Gene Bio-tech Co. Ltd., Shanghai, China). The analyses of the cDNA and amino acid sequences of EtMIC2 were carried out as previously described [14]. Briefly, the molecular mass was obtained using translate tool software at the ExPASy server of the Swiss Institute of Bioinformatics (http://www.expasy.org/tools/ protparam.html). The signal peptide and transmembrane (TM) regions were predicted using SignalP (http://www.cbs.dtu.dk/ services/SignalP/) and TMHMM (http://www.cbs.dtu.dk/ services/TMHMM-2.0/), respectively.

\section{Expression and purification of recombinant EtMIC2}

The EtMIC2 gene was then cloned into the pET32a(+) vector (Novagen, Merck KGaA, Darmstadt, Germany) using the EcoRI and HindIII sites. The recombinant plasmid was confirmed by DNA sequencing and transformed into the 
E. coli BL21 (DE3) (Promega) expression strain. The expression of rEtMIC2 in E. coli was induced using $0.8 \mathrm{mM}$ isopropyl- $\beta$-D-thiogalactopyranoside (IPTG, Sigma-Aldrich, St. Louis, MO, USA) at $37^{\circ} \mathrm{C}$ for $8 \mathrm{~h}$. The induced bacterial cells were collected by centrifugation and sonicated on ice. The supernatant was collected, and the recombinant protein was purified using a His Bind Purification kit (Novagen). The purified protein was analyzed with $12 \%$ sodium dodecyl sulfate polyacrylamide gel electrophoresis and its concentration determined using a BCA protein assay kit (Novagen). The purified protein was then stored in aliquots at $-20{ }^{\circ} \mathrm{C}$ until further use [7].

\section{Production of polyclonal sera against recombinant EtMIC2}

Two-month-old rabbits were immunized subcutaneously with $0.2 \mathrm{mg}$ of purified rEtMIC2 emulsified in an equal volume of Freund's complete adjuvant (Sigma-Aldrich). This was followed by three booster injections with rEtMIC2 emulsified in an equal volume of Freund's incomplete adjuvant (Sigma-Aldrich) at two-week intervals. Seven days after the final immunization, serum was separated from rabbit blood. Serum collected before immunization was used as the negative control. The anti-sera were then stored at $-80{ }^{\circ} \mathrm{C}$ until further use.

\section{Immunolocalization of EtMIC2 in parasites with an indirect immunofluorescence assay}

The localization of the EtMIC2 protein was investigated using an immunofluorescence assay (IFA), as previously described [14], with slight modifications. In brief, DF-1 cells $\left(3 \times 10^{5}\right.$ cells per well) were seeded in six-well plates (Corning, Corning, NY, USA) with pre-coated sterile coverslips and cultured in complete medium (DMEM containing 10\% FBS, $100 \mathrm{U} / \mathrm{mL}$ penicillin/streptomycin, $2 \mathrm{mM}$ L-glutamine) at $37{ }^{\circ} \mathrm{C}$ in $5 \% \mathrm{CO}_{2}$ for $24 \mathrm{~h}$. Freshly excysted sporozoites were incubated for $1 \mathrm{~h}$ at $41{ }^{\circ} \mathrm{C}$ in complete medium. Then the sporozoites were added to adherent cells at a ratio of one sporozoite per cell. Infected DF-1 cells were collected at $72 \mathrm{~h}$ post infection for fixation. Sporozoites in infected DF-1 cells were washed with phosphate buffered saline (PBS) for $10 \mathrm{~min}$, fixed in $2 \%$ paraformaldehyde for $20 \mathrm{~min}$, permeabilized with $1 \%$ Triton-X in PBS for $15 \mathrm{~min}$, and then blocked with $2 \%$ bovine serum albumin in PBS overnight at $4{ }^{\circ} \mathrm{C}$. The coverslips were then incubated with a rabbit anti-rEtMIC2 antibody $(1: 100$ dilution) for $1 \mathrm{~h}$ at $37{ }^{\circ} \mathrm{C}$, and then further incubated for $1 \mathrm{~h}$ with goat anti-rabbit $\operatorname{IgG}$ fluorescein isothiocyanate (FITC)-conjugated antibody (1:500 dilution; Sigma-Aldrich) in a moist, dark chamber. Cell nuclei were labelled with $10 \mu \mathrm{g} / \mathrm{mL} 4$, 6-diamidino-2-phenylindole (DAPI, Beyotime, Haimen, China) for $10 \mathrm{~min}$. The coverslips were washed three times in PBS with $0.5 \%$ Triton-X 100 after every step. The coverslips were finally mounted on glass slides using $60 \mu \mathrm{L}$ of Fluoromount Aqueous Mounting Medium (Sigma-Aldrich) and viewed under a fluorescence microscope (Nikon, Tokyo, Japan). Sporozoites and second-generation merozoites were prepared for immunofluorescence observation using the same method.

\section{Sporozoite invasion inhibition assay}

The invasion inhibition assay was based on the observation that E. tenella sporozoites invade DF-1 cells [12, 13]. All antibodies were purified using Protein $\mathrm{A}+\mathrm{G}$ agarose (Beyotime), according to the manufacturer's instructions, and their concentration was determined using a BCA protein assay kit (Novagen). DF- 1 cells $\left(3 \times 10^{5}\right.$ cells per well) were seeded in 24-well plates and cultured in DMEM with 10\% FBS at $37{ }^{\circ} \mathrm{C}$ in $5 \% \mathrm{CO}_{2}$ for $24 \mathrm{~h}$. Freshly isolated sporozoites were labelled with the dye carboxyfluorescein diacetate succinimidyl ester (CFSE-Molecular Probes, Beyotime), according to the manufacturer's instructions, and incubated at $37^{\circ} \mathrm{C}$ with either $100,200,300$, or $400 \mu \mathrm{g} / \mathrm{mL}$ of purified IgG against rEtMIC2 for $2 \mathrm{~h}$. The same quantity of purified IgG from naive rabbit sera was used as a control. The sporozoite- $\operatorname{IgG}$ mixture was centrifuged at $2000 \times g$ for $5 \mathrm{~min}$. The pellet was then resuspended in DMEM containing $10 \%$ FBS, penicillin $(100 \mathrm{U} / \mathrm{mL})$ and streptomycin $(100 \mu \mathrm{g} / \mathrm{mL})$. Following this, the pre-treated sporozoites were used to infect adherent DF-1 cells at a ratio of one sporozoite per cell and then cultured at $41{ }^{\circ} \mathrm{C}$ in $5 \% \mathrm{CO}_{2}$ for $12 \mathrm{~h}$. Cells were washed twice in sterile PBS and then trypsinized, collected, and analyzed with Cytomics FC500 flow cytometry (Beckman Coulter Inc., Fullerton, CA, USA). All assays were performed in triplicate. The percentage of infected cells in the presence or absence of the inhibitory antibody was used to calculate inhibition rates, as previously described [12].

\section{Construction of the pcDNA3.1(+)-EtMIC2 DNA vaccine and its expression in vitro}

The EtMIC2 gene was cloned into the pcDNA3.1(+) vector using the BamHI/HindIII sites. The recombinant plasmid was then sequenced at Invitrogen Biotech (Shanghai, China) to confirm that the EtMIC2 gene was inserted into the correct open reading frame. The recombinant plasmid pcDNA3.1 $(+)$-EtMIC2, and the empty plasmid pcDNA3.1(+) were prepared as a DNA vaccine using the Qiagen EndoFree Plasmid Kit (Qiagen Biotech, Beijing, China), following the manufacturer's instructions. The eluted product was dissolved in $10 \mathrm{mM}$ Tris- $\mathrm{HCl} \mathrm{pH} 8.0$ and $1 \mathrm{mM}$ EDTA (TE) buffer at $1 \mathrm{mg} / \mathrm{mL}$ and stored at $-20{ }^{\circ} \mathrm{C}$ until required.

DF-1 cells were grown in six-well plates until a confluency of $80 \%-90 \%$. The cells were then transfected with $4 \mu \mathrm{g}$ of pcDNA3.1(+)-EtMIC2 or the empty plasmid pcDNA3.1(+) using Lipofectamine 2000 (Invitrogen), as per the manufacturer's instructions. Briefly, the transfection reagent and recombinant plasmid were mixed $(10 \mathrm{~mL}$ Lipofectamine 2000 and $4 \mu \mathrm{g}$ DNA), incubated at room temperature for $30 \mathrm{~min}$, and then added to the cells. Six hours later, the DNA-transfection reagent mixture was replaced with DMEM containing 10\% FBS. After $48 \mathrm{~h}$ post-transfection, the expression of the EtMIC2 protein encoded in the transfected plasmid was confirmed by indirect IFA with antibodies against EtMIC2 [7]. 
A

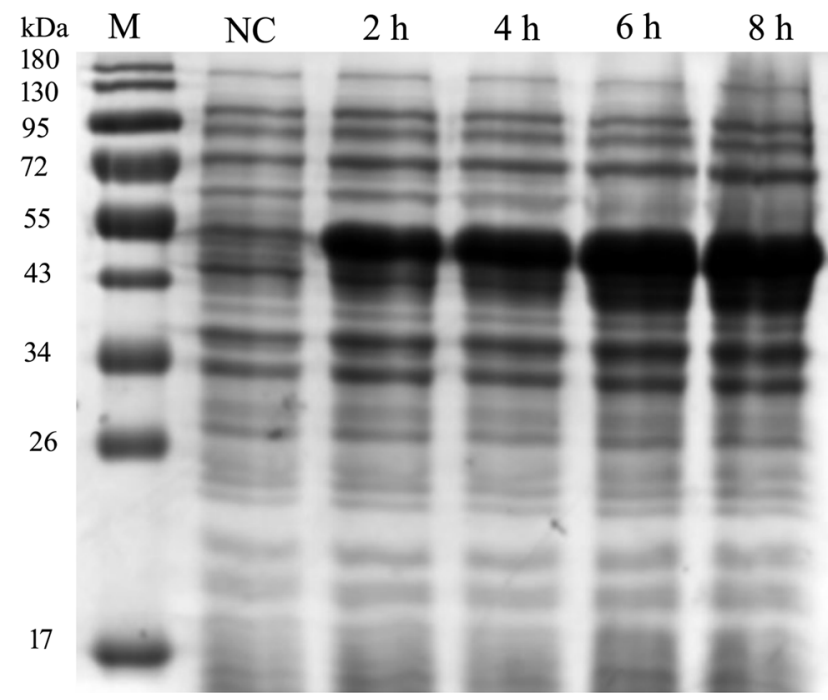

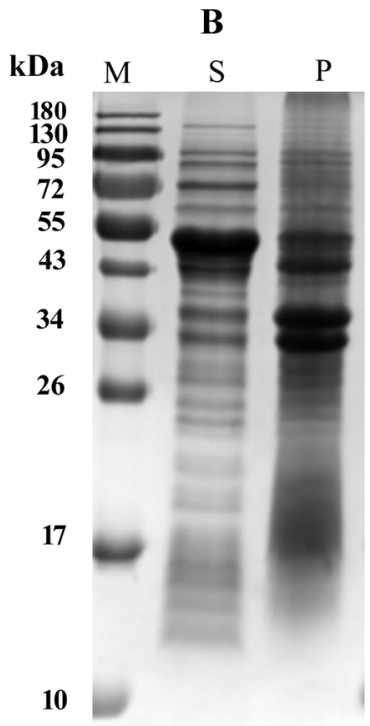

Figure 1. Analysis of EtMIC2 expression in E. coli BL21. (A) Protein expression in bacterial pellets at different times of induction. Lane 1 protein marker; Lane 2 negative control (not induced with IPTG); Lanes 3, 4, 5, and 6 induced with IPTG at 2, 4, 6, and 8 h, respectively. (B) Protein expression in sonicated bacterial cells induced with IPTG at $8 \mathrm{~h}$. S: supernatant, P: precipitate.

\section{Immunization and parasite-challenge infection}

One-day-old chickens were housed in a clean house and at the age of seven days, chickens were randomly selected, weighed, and divided into four groups, each consisting of 20 chickens. Experimental groups were inoculated with $100 \mu \mathrm{g}$ of either pcDNA3.1(+)-EtMIC2 or empty vector pcDNA3.1(+) by intramuscular injection. Chickens in the challenged control and non-challenged control groups were injected with the same TE buffer at the same site of injection. Seven days after the first vaccination, a booster vaccination was performed as before. Seven days after the booster vaccination, all chickens were infected orally with 10,000 freshly sporulated E. tenella oocysts, except for the non-challenged control group, which were inoculated with PBS. All chickens were humanely killed eight days post-challenge to evaluate the lesion score, as previously described [15]. Chicken body weights were measured at day 0 and 8 post-challenge. Feces from each group were collected separately at day 6-8 post-challenge. Oocyst shedding per bird was determined using a McMaster chamber $[18,36]$. Each fecal sample was counted three times using the same method. The oocyst decrease ratio (\%) was calculated as follows: the average number of oocysts per bird from the challenged control group - the average number of oocysts per bird from vaccinated group/the average number of oocysts per bird from the challenged control group $\times 100$.

\section{Statistical analysis}

All data were subjected to the software SPSS version 20.0 for Windows (IBM, Armonk, NY, USA) for analysis. The Shapiro-Wilk test was performed to assess normality for body weight gains $(p>0.05)$ and oocyst shedding per bird $(p>0.05)$. The significance of differences in body weight gains and oocyst shedding per bird among the groups were evaluated by one-way analysis of variance (ANOVA) and mean values were compared using Duncan's multiple range tests. The lesion scores were compared by the nonparametric Kruskal-Wallis test. The difference was considered significant if $p<0.05$.

\section{Results \\ Cloning of the EtMIC2 gene}

The EtMIC2 gene was isolated from cDNA of sporulated oocysts from the E. tenella Shanghai strain. After cloning and sequencing, a predicted 1127 base pair product was obtained and analyzed with BLASTn. The sequence displayed 100\% identity with the known MIC2 gene from the E. tenella Beijing strain (GenBank: AF111839.1), suggesting that the EtMIC2 gene from the E. tenella Shanghai strain had been successfully amplified. Furthermore, the protein showed a $100 \%, 99.7 \%$ and $99.7 \%$ identity with the MIC2 from the E. tenella Beijing strain (AAD05559), Houghton strain (XP_013233366.1) and India strain (ACN93990.1), respectively from the GenBank database. Sequence analysis of the EtMIC2 open reading frame identified a polypeptide consisting of 342 amino acid residues with a predicted molecular mass of $35.07 \mathrm{kDa}$. In addition, the amino acid sequence showed no signs of a transmembrane region; however, it had a signal peptide (residues 1-21).

\section{Expression and purification of recombinant EtMIC2 protein}

rEtMIC2 was successfully expressed in both the soluble and insoluble fraction of $E$. coli after induction with $0.8 \mathrm{mM}$ IPTG at $37{ }^{\circ} \mathrm{C}$ for $8 \mathrm{~h}$. Purified protein was obtained from the supernatant only using a His Bind Purification kit. The molecular mass of the rEtMIC2 fused to the His-tag was found to be approximately $53 \mathrm{kDa}$, as expected (Fig. 1). 


\section{A: sporozoites}

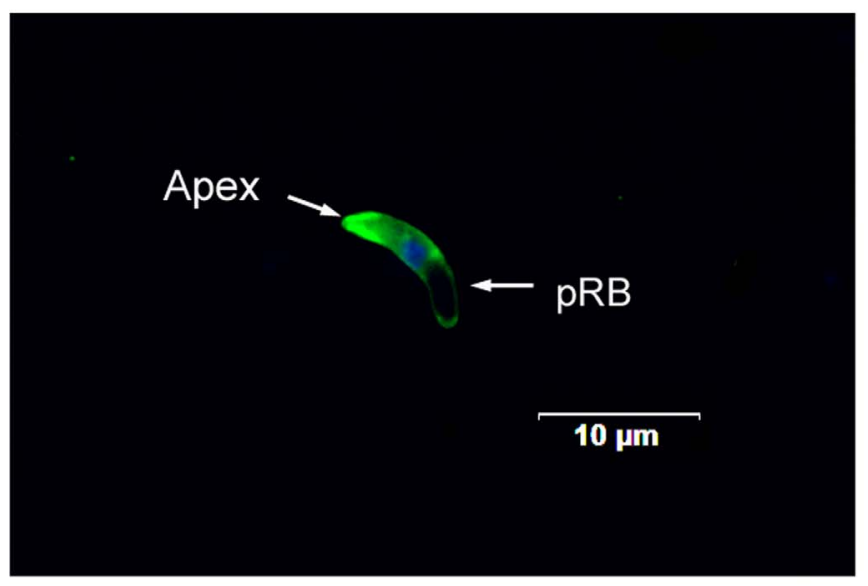

B: second-generation merozoites

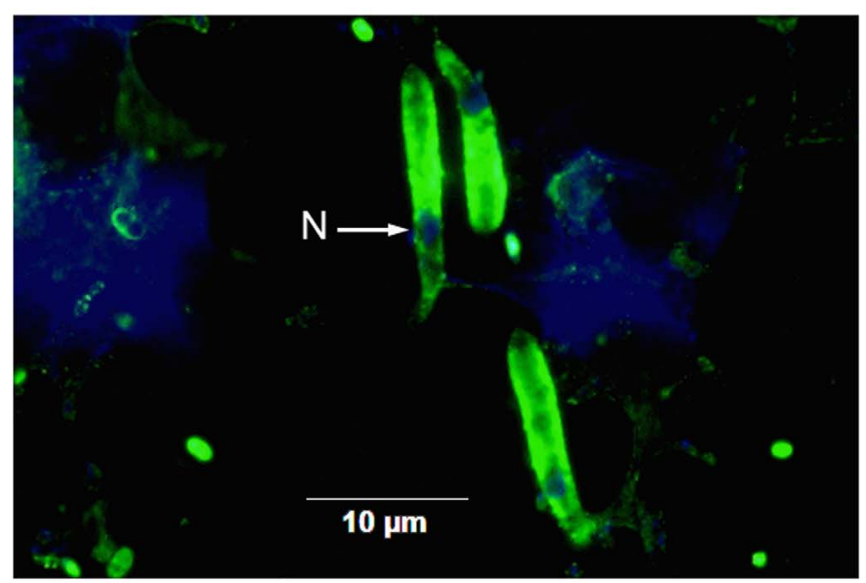

\section{C: mature first-generation schizonts}

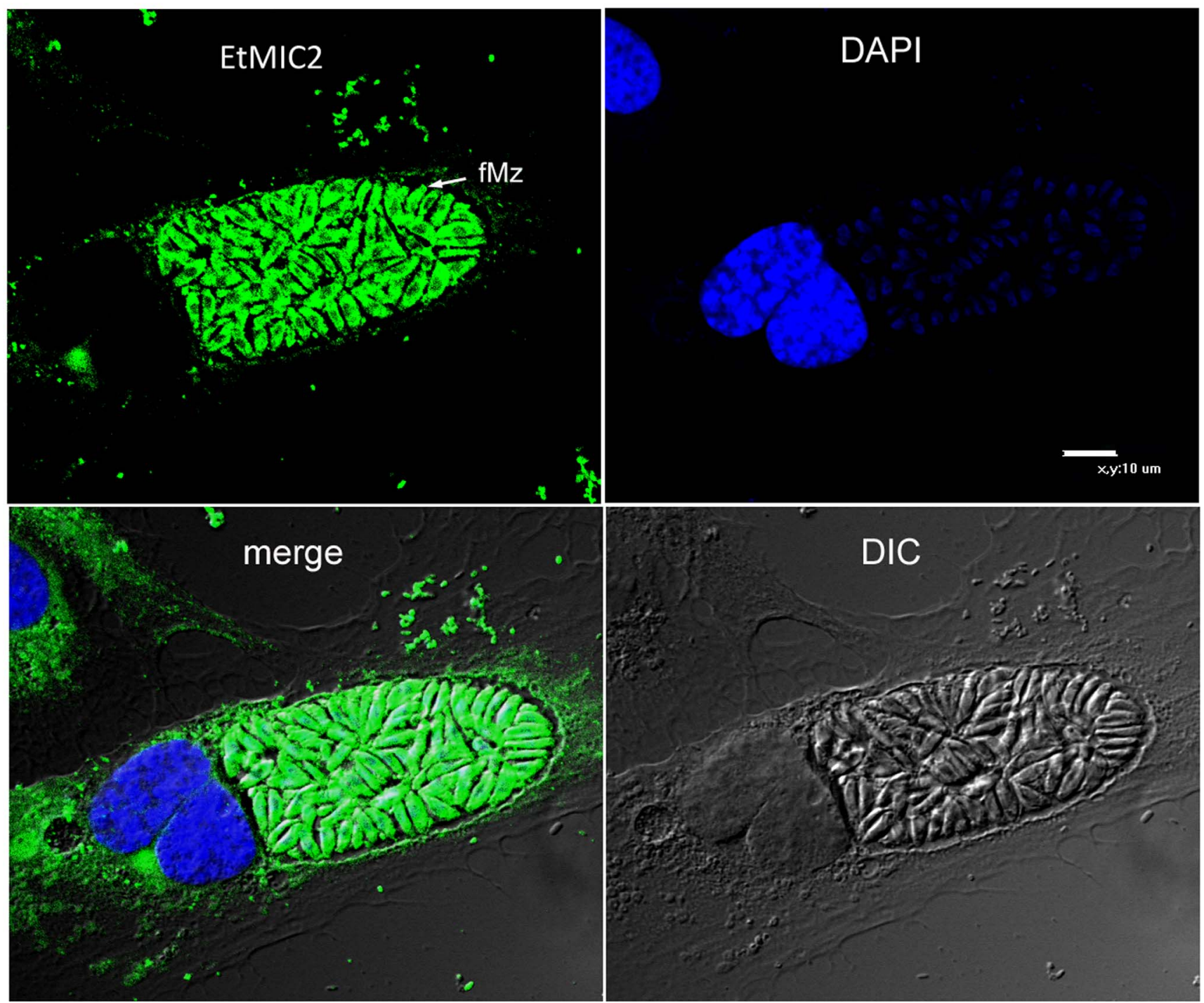

Figure 2. Localization of EtMIC2 in sporozoites, second-generation merozoites, and mature first-generation schizonts by indirect immunofluorescence. The parasites were immunostained with anti-rEtMIC2 antibodies, visualized with FITC (green) and counter-stained with DAPI (blue). Abbreviations: pRB, posterior refractile body; $\mathrm{N}$, nucleus; fMz, first-generation merozoites. In vitro sporozoite inhibition assays using recombinant EtMIC2 anti-sera. 


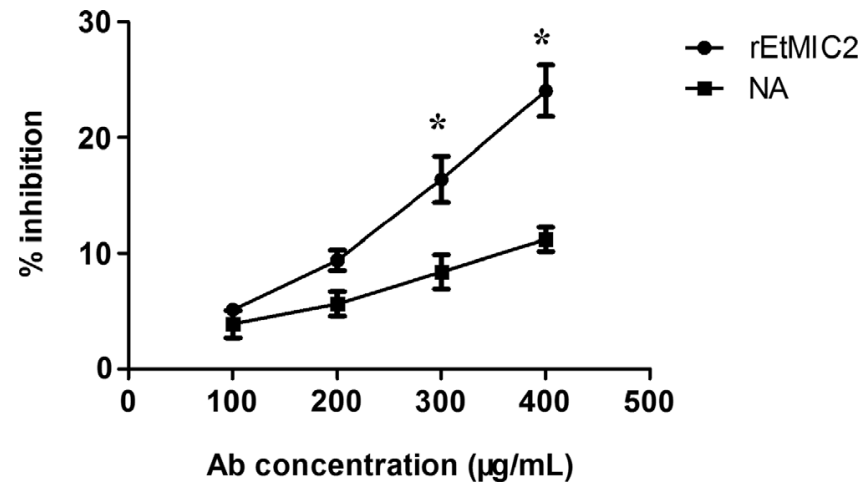

Figure 3. In vitro sporozoite inhibition assays using recombinant EtMIC2 anti-sera. Anti-rEtMIC2 stands for IgG purified from recombinant EtMIC2 anti-sera. NA stands for IgG from naive rabbit serum. All assays were performed in triplicate. $(*)$ Indicates that differences between the treatment with anti-rEtMIC 2 antibodies and with naive rabbit serum at the same IgG concentration were significant $(p<0.05)$.

\section{Immunofluorescence localization of EtMIC2 in parasites}

The localization of EtMIC2 in sporozoites, second generation merozoites, and mature first-generation schizonts was investigated using an indirect IFA with anti-rEtMIC2 as a probe. Labeled EtMIC2 was found to mainly locate in the anterior region and membrane of newly excysted sporozoites (Fig. 2A). Furthermore, the EtMIC2 protein was found to be strongly expressed in mature first-generation schizonts, and was concentrated in the cytoplasm of first- and secondgeneration merozoites (Figs. 2B and 2C).

\section{Inhibition of $E$. tenella invasion by antibodies against rEtMIC2}

An invasion inhibition assay was performed to test the ability of rabbit anti-rEtMIC2 antibodies to inhibit the invasion of DF-1 cells by E. tenella sporozoites. It was found that the inhibition effect was in fact dose-dependent (Fig. 3). As compared with the same dose of naive rabbit sera IgG (used as a negative control), pretreatment with 100 or $200 \mu \mathrm{g} / \mathrm{mL}$ antiEtMIC2 IgG did not significantly affect the invasion capacity of sporozoites $(p>0.05)$; however, pretreatment with 300 and $400 \mu \mathrm{g} / \mathrm{mL}$ did significantly decrease the invasion capacity of $E$. tenella sporozoites $(p<0.05)$.

\section{Immunofluorescence assay detection of eukaryotic plasmids in DF-1 cells}

Expression of the recombinant plasmid pcDNA3.1(+)EtMIC2 and the negative control plasmid pcDNA3.1(+) was confirmed by IFA after transfection with DF-1 cells for $48 \mathrm{~h}$. The intense green fluorescence was detected in DF-1 cells transfected with pcDNA3.1(+)-EtMIC2 and none was in those transfected with pcDNA3.1(+) (Fig. 4), demonstrating that the pcDNA3.1(+)-EtMIC2 protein could be successfully expressed in vitro.

\section{Protective efficacy of pcDNA3.1(+)-EtMIC2 vaccination against $E$. tenella in chickens}

The efficacies of the current immunization and challenge assay were evaluated on the basis of body weight gain, oocyst shedding, and the cecal lesion score (Table 1). After challenge, chickens vaccinated with pcDNA3.1(+)-EtMIC2 gained significantly more body weight and had significantly fewer cecal lesions and oocysts compared with chickens vaccinated with the empty vector pcDNA3.1(+) or the TE-challenged controls. Additionally, oocyst shedding in the pcDNA3.1(+) group was significantly lower than in the TE-challenged control groups, but there were no significant differences in terms of body weight and cecal lesions between the two control groups.

\section{Discussion}

In this study, the EtMIC2 gene from E. tenella Shanghai strain was cloned and characterized. The obtained sequence showed $99 \%-100 \%$ identity with the available EtMIC2 genes deposited in GenBank, indicating that the EtMIC2 gene is highly conserved among the different strains of E. tenella [37]. Furthermore, sequence analysis found that the EtMIC2 gene predicted a protein with a classical signal peptide at the mature N-terminus of the protein. Interestingly, regions of the EtMIC2 protein have previously been found to have highly significant similarities to regions within Drosophila melanogaster tropomyosin II and within two substrates of the cellular regulatory enzyme protein kinase C [39].

EtMIC2 is an acidic protein that is abundant within the microneme organelles. Several studies have previously indicated that EtMIC2 forms a complex with EtMIC1, and this complex presumably becomes mobilized from micronemes to the parasite surface during attachment and is then redistributed towards the posterior end of the parasite during penetration of the host cell [39]. Using an antibody raised against the rEtMIC2, immunolocalization studies in E. tenella showed that the protein was mainly located in the anterior region and membrane of E. tenella sporozoites. Furthermore, the EtMIC2 protein was strongly expressed during first schizogony and was mainly located in the cytoplasm of first- and second-generation merozoites. These results are consistent with previous studies where a monoclonal antibody (mAb) specific to EtMIC2 was used to detect the MIC2 location in several Eimeria spp., including E. tenella, E. mitis, E. stiedai, and E. irresidua, finding that MIC2 was located in the anterior region of the sporozoites among all Eimeria spp. [38]. Additionally, Sasai et al [28] found that the EtMIC2 mAb primarily stained a small region of the apical tip of the sporozoites and merozoites from E. tenella, E. acervulina, and E. maxima. However, there were some differences in the observed staining patterns between the three species. In E. tenella and E. maxima, there also appeared to be staining of the basal anterior tip [28]. But in E. acervulina, a distinct cone-like morphology was stained on the anterior tip of sporozoites and merozoites, with little staining in other regions [28].

Although micronemal in origin, the EtMIC2 protein has been clearly shown to translocate to the surface of the 

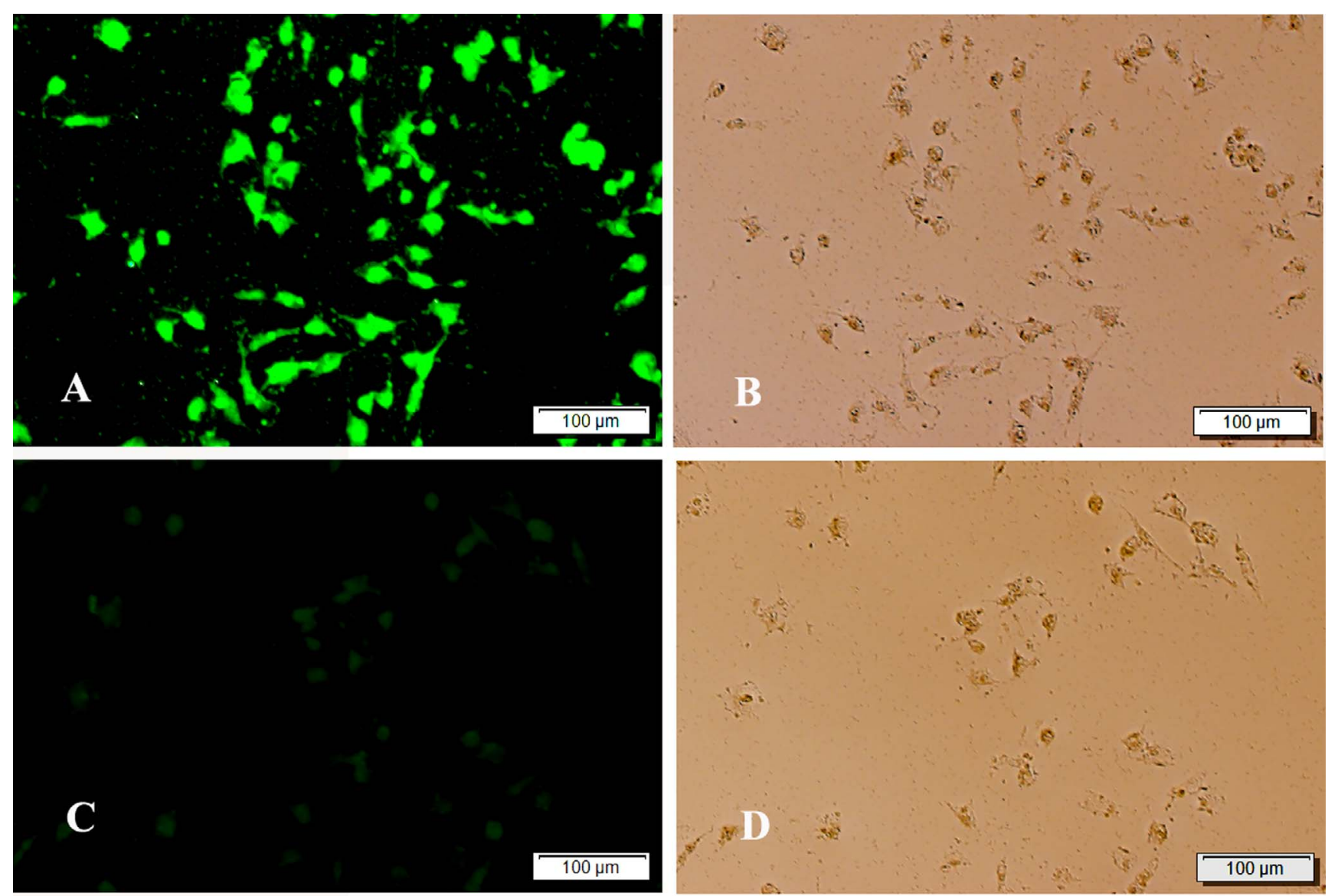

Figure 4. Indirect immunofluorescence assay of EtMIC2 in transfected DF-1 cells. (A, B) pcDNA3.1(+)-EtMIC2-transfected DF-1 cells under fluorescent (FITC) illumination and brightfield, respectively. (C, D) pcDNA3.1(+)-transfected DF-1 cells under fluorescent illumination and brightfield, respectively.

Table 1. Protective effects of pcDNA3.1(+)-EtMIC2 against experimental Eimeria tenella infection in chickens.

\begin{tabular}{lccccc}
\hline Groups & $\begin{array}{c}\text { Average body } \\
\text { weight gains }(\mathrm{g})\end{array}$ & $\begin{array}{c}\text { Reduced rate of } \\
\text { weight gain }(\%)\end{array}$ & $\begin{array}{c}\text { Oocyst shedding } \\
\text { per bird }\left(10^{7}\right)\end{array}$ & $\begin{array}{c}\text { Oocyst decrease } \\
\text { ratio }(\%)\end{array}$ & $\begin{array}{c}\text { Lesion scores } \\
\text { pcDNA3.1(+)-EtMIC2 }\end{array}$ \\
pcDNA3.1(+) & $132.88 \pm 38.06^{\mathrm{a}}$ & 5.44 & $2.09 \pm 0.17^{\mathrm{b}}$ & 75.30 & $0.53 \pm 0.04^{\mathrm{b}}$ \\
TE-infected & $122.61 \pm 33.41^{\mathrm{b}}$ & 12.75 & $6.17 \pm 0.25^{\mathrm{c}}$ & 27.07 & $1.54 \pm 0.07^{\mathrm{c}}$ \\
TE-uninfected & $118.61 \pm 24.40^{\mathrm{b}}$ & 15.6 & $8.46 \pm 0.24^{\mathrm{d}}$ & 0 & $1.58 \pm 0.08^{\mathrm{c}}$ \\
\hline
\end{tabular}

Values are expressed as mean \pm standard deviation $(S D)$. Means in the same column with different letters were found to be significantly different between treatment groups $(p<0.05)$.

sporozoite, and then during invasion of host cells the protein concentrated around the point of parasite entry, secreted into the extracellular milieu and transferred to the surface of the infected host cell where it stained in uneven patches [39]. These results suggest that EtMIC2 may be involved in cell invasion, but this needs to be confirmed through an invasion assay. Previously, it has been shown that invasion inhibition assays in vitro clearly reduced sporozoite invasion in the presence of an mAb or specific polyclonal antibody [12, 13, 24]. In the current study, such assays showed that pretreatments with 300 and $400 \mu \mathrm{g} / \mathrm{mL}$ rEtMIC2-specific antibody, with inhibition rates of $16.41 \%$ and $24.06 \%$, respectively, significantly reduced sporozoite penetration of cultured cells. These data therefore confirm that EtMIC2 plays an important role in host invasion.

The lifecycle of $E$. tenella involves endogenous (schizogony and gametogony) and exogenous (sporogony) stages, and the identification of genes expressed in the lifecycle of coccidia is critical in understanding the developmental biology of these parasites. Previously, it has been shown that EtMIC2 is an important microneme protein, expressed abundantly in the sporozoite and schizogony stages [3, 26, 28, 39]. Furthermore, Liu et al. detected the expression of EtMIC2 in the sexual developmental stages of gametocytes 
and zygotes in chickens artificially infected with E. tenella using immunostaining and western blot analysis with a monoclonal anti-EtMIC2 antibody [20]. These results suggest that the protein is actually expressed abundantly in all endogenous developmental stages of E. tenella and would be an ideal candidate for vaccine development [47].

Rotational treatment with anticoccidial drugs and a commercial live vaccine is currently the best way to control infection in chicken flocks. Due to the high expense of scaling-up the production of the live parasite vaccine, there have been a number of recent efforts to develop subunit and recombinant coccidiosis vaccines using both DNA and protein-based antigens $[16,21,30]$. However, few have been successful and much more work is needed to identify appropriate antigens and the optimal mode of delivery [17]. The EtMIC2 protein has been found to be conserved in the parasite and its role in the early stages of invasion suggests that it may serve as an effective vaccine antigen. The EtMIC2 gene or rEtMIC2 proteins expressed in prokaryotic, plant or Pichia pastoris expression systems have been used as either a DNA vaccine or sub-unit vaccine to immunize chickens against a homologous challenge in several early studies [6, 29, 45, 46]. Taken together, these studies suggest that EtMIC2 can provide partial protection against a challenge, although the protective levels were found to be different between each of these studies. In this study, a recombinant chimeric subunit vaccine was generated, consisting of EtMIC2 and a eukaryotic expression vector, and its efficacy against $E$. tenella infection in chickens was evaluated. The eukaryotic expression vector used in this study pcDNA3.1(+), has been widely used in the development of DNA vaccines against coccidiosis [19, 43, 44]. EtMIC2 protein expression was confirmed with an in vitro method before carrying out in vivo experiments. Intense fluorescence in DF-1 cells transfected with pcDNA3.1(+)-EtMIC2 indicated that the recombinant plasmid pcDNA3.1(+)-EtMIC2 was successfully constructed and expressed in the eukaryotic cells. Furthermore, the results of the challenge experiments showed that chickens treated with the DNA vaccine gained significantly more weight, and had significantly fewer cecal lesions and oocysts, compared with infected chickens treated with the control vaccine. In the present study, the number of oocysts produced per oocyst administered (the "reproductive potential") was much lower than that from Williams [42], because a challenge dose higher than the crowding threshold of E. tenella was used and parasite replication cannot be accurately assessed due to the crowding effect. In order to minimize this limitation, the relative oocyst decrease ratio [= (oocyst number from challenged unvaccinated group - oocyst number from challenged vaccinated group)/oocysts number from challenged unvaccinated group $\times 100$ ], instead of the oocyst production, together with weight gains and cecal lesion scores, were used to evaluate the protective efficacy of this DNA vaccine. While these are small-scale experiments, the consistency of the trial and level of efficacy with an approximate reduction of $\sim 75.30 \%$ in oocyst shedding following vaccination, being higher than what has been previously seen in many studies with other antigens, indicates that EtMIC2 should be considered as an ideal candidate antigen in the development of a new vaccine against $E$. tenella in chickens.
In summary, EtMIC2 was found to be located in the anterior region and membrane of sporozoites, was strongly expressed during first schizogony, and was mainly located in the cytoplasm of first- and second-generation merozoites. Additionally, rEtMIC2-specific antibody was shown to inhibit parasite invasion. The recombinant plasmid pcDNA3.1(+)EtMIC2 induced partial protective immunity in immunized chickens. Overall, these results suggest that EtMIC2 may play an important role in parasite cell invasion and may be an ideal candidate for the development of new vaccines against E. tenella infection in chickens

\section{Competing interest}

The authors declare that there are no competing interests.

Acknowledgements. This work was supported by grants from the National Science Foundation of China (Nos. 31672551, 31572266), National Key R\&D program of China (No. 2017YFD0500400), and National Sharing Service Platform for Parasite Resource (No. TDRC-22).

\section{References}

1. Blake DP, Tomley FM. 2014. Securing poultry production from the ever-present Eimeria challenge. Trends in Parasitology, 30, 12-19.

2. Bromley E, Leeds N, Clark J, McGregor E, Ward M, Dunn MJ, Tomley FM. 2003. Defining the protein repertoire of microneme secretory organelles in the apicomplexan parasite Eimeria tenella. Proteomics, 3(8), 1553-1561.

3. Bumstead J, Tomley FM. 2000. Induction of secretion and surface capping of microneme proteins in Eimeria tenella. Molecular and Biochemical Parasitology, 110(2), 311-321.

4. Chapman HD, Barta JR, Blak D, Gruber A, Jenkins M, Smith NC, Suo X, Tomley FM. 2013. A selective review of advances in coccidiosis research. Advances in Parasitology, 83, 93-171.

5. Chapman HD. 1997. Biochemical, genetic and applied aspects of drug resistance in Eimeria parasites of the fowl. Avian Pathology, 26, 221-244.

6. Ding X, Lillehoj HS, Dalloul RA, Min W, Sato T, Yasuda A, Lillehoj EP. 2005. In ovo vaccination with the Eimeria tenella EtMIC2 gene induces protective immunity against coccidiosis. Vaccine, 23, 3733-3740.

7. Dong H, Yang S, Zhao Q, Han H, Zhu S, Zhu X, Li C, Wang Z, Xia W, Men Q, Yang LY. 2016. Huang B. Molecular characterization and protective efficacy of silent information regulator 2a from Eimeria tenella. Parasite \& Vectors, 9, 602.

8. Han H, Dong H, Zhu S, Zhao Q, Jiang L, Wang Y, Li L, Wu Y, Huang B. 2014. Molecular characterization and analysis of a novel protein disulfide isomerase-like protein of Eimeria tenella. PLoS One, 9, e99914.

9. Han H, Xue P, Dong H, Zhu S, Zhao Q, Huang B. 2016. Screening and characterization of apical membrane antigen 1 interacting proteins in Eimeria tenella. Experimental Parasitology, 170, 116-124.

10. Huang B, Zhao QP, Wu XZ, Shi TW, Chen ZG. 1993. Study on the identification and pathogenicity of the pure species of Eimeria tenella. Shanghai Journal of Animal Husbandry and Veterinary Medicine, 3, 18-20 (in Chinese).

11. Huynh MH, Opitz C, Kwok LY, Tomley FM, Carruthers VB, Soldati D. 2004. Trans-genera reconstitution and 
complementation of an adhesion complex in Toxoplasma gondii. Cellular Microbiology, 6, 771-782.

12. Jahn D, Matros A, Bakulina AY, Tiedemann J, Schubert U, Giersberg M, Haehnel S, Zoufal K, Mock HP, Kipriyanov SM. 2009. Model structure of the immunodominant surface antigen of Eimeria tenella identified as a target for sporozoiteneutralizing monoclonal antibody. Parasitology Research, 105, 655-668.

13. Jiang LL, Lin JJ, Han HY, Dong H, Zhao QP, Zhu SH, Huang B. 2012. Identification and characterization of Eimeria tenella apical membrane antigen-1(AMA1). PLoS One, 7, e41115.

14. Jiang LL, Lin JJ, Han HY, Zhao QP, Dong H, Zhu SH, Huang B. 2011. Identification and partial characterization of a serine protease inhibitor (serpin) of Eimeria tenella. Parasitology Research, 110, 865-874.

15. Johnson J, Reid WM. 1970. Anticoccidial drug: lesion scoring techniques in battery and floor-pen experiments with chickens. Experimental Parasitology, 28, 30-36.

16. Klotz C, Gehre F, Lucius R, Pogonka T. 2007. Identification of Eimeria tenella genes encoding for secretory proteins and evaluation of candidates by DNA immunisation studies in chickens. Vaccine, 25, 6625-6634.

17. Lai L, Bumstead J, Liu Y, Garnett J, Campanero-Rhodes MA, Blake DP, Palma AS, Chai W, Ferguson DJ, Simpson P, Feizi T, Tomley FM, Matthews S. 2011. The role of sialyl glycan recognition in host tissue tropism of the avian parasite Eimeria tenella. PLoS Pathogens, 7(10), e1002296.

18. Lee SH, Lillehoj H, Dalloul RA, Park DW, Hong YH, Lin JJ. 2007. Influence of Pediococcus-based probiotic on coccidiosis in broiler chickens. Poultry Science, 86, 63-66.

19. Lillehoj HS, Ding X, Quiroz MA, Bevensee E, Lillehoj EP. 2005. Resistance to intestinal coccidiosis following DNA immunization with the cloned 3-1E Eimeria gene plus IL-2, IL-15, and IFN- $\gamma$. Avian Disease, 49, 112-117.

20. Liu Q, Chen Z, Shi W, Sun H, Zhang J, Li H, Xiao Y, Wang F, Zhao X. 2014. Preparation and initial application of monoclonal antibodies that recognize Eimeria tenella microneme proteins 1 and 2. Parasitology Research, 113, 4151-4161.

21. Ma D, Ma C, Pan L, Li G, Yang J, Hong J, Cai H, Ren X. 2011. Vaccination of chickens with DNA vaccine encoding Eimeria acervulina 3-1E and chicken IL-15 offers protection against homologous challenge. Experimental Parasitology, 127, 208-214.

22. Morahan BJ, Wang L, Coppel RL. 2009. No TRAP, no invasion. Trends in Parasitology, 25(2), 77-84.

23. Peek HW, Landman WJ. 2011. Coccidiosis in poultry: Anticoccidial products, vaccines and other prevention strategies. Veterinary Quarterly, 31, 143-161.

24. Peroval M, Pery P, Labbe M. 2006. The heat shock protein 90 of Eimeria tenella is essential for invasion of host cell and schizont growth. International Journal for Parasitology, 36, 1205-1215.

25. Rabenau KE, Sohrabi A, Tripathy A, Reitter C, Ajioka JW, Tomley FM, Carruthers VB. 2001. TgM2AP participates in Toxoplasma gondii invasion of host cells and is tightly associated with the adhesive protein TgMIC2. Molecular Microbiology, 41(3), 537-547.

26. Ryan R, Shirley M, Tomley F. 2000. Mapping and expression of microneme genes in Eimeria tenella. International Journal for Parasitology, 30, 1493-1499.

27. Sam-Yellowe T. 1996. Rhoptry organelles of the apicomplexa: Their role in host cell invasion and intracellular survival. Parasitology Today, 12(8), 308-316.
28. Sasai K, Fetterer RH, Lillehoj H, Matsuura S, Constantinoiu CC, Matsubayashi M, Tani H, Baba E. 2008. Characterization of monoclonal antibodies that recognize the Eimeria tenella microneme protein MIC2. Journal of Parasitology, 94 1432-1434

29. Sathish K, Sriraman R, Subramanian BM, Rao NH, Balaji K, Narasu ML, Srinivasan VA. 2011. Plant expressed MtMIC2 is an effective immunogen in conferring protection against chicken coccidiosis. Vaccine, 29, 9201-9208.

30. Shah MAA, Yan R, Xu L, Song X, Li X. 2010. A recombinant DNA vaccine encoding Eimeria acervulina cSZ-2 induces immunity against experimental E. tenella infection. Veterinary Parasitology, 169, 185-189.

31. Sharman PA, Smith NC, Wallach MG, Katrib M. 2010. Chasing the golden egg: Vaccination against poultry coccidiosis. Parasite Immunology, 32, 590-598.

32. Shi W, Liu Q, Zhang J, Sun J, Jiang X, Geng J, Wang F, Xiao Y, Li H, Zhao X. 2014. Co-expression of EtMic2 protein and chicken interleukin-18 for DNA vaccine against chicken coccidiosis. Research in Veterinary Science, 97, 64-70.

33. Shirley MW, Smith AL, Blake DP. 2007. Challenges in the successful control of the avian coccidia. Vaccine, 25, 5540-5547.

34. Shirley MW. 1995. Eimeria species and strains of chickens, in Biotechnology guidelines on techniques in coccidiosis research. Coudert P, Eckert J, Braun R, Shirley MW, Editors. The European Commission DGXII: Luxembourg City, Luxembourg. p. 9-10.

35. Sonda S, Fuchs N, Gottstein B, Hemphill A. 2000. Molecular characterization of a novel microneme antigen in Neospora caninum. Molecular and Biochemical Parasitology, 108(1), 39-51.

36. Sun H, Wang L, Wang T, Zhang J, Liu Q, Chen P, Chen Z, Wang F, Li H, Xiao Y, Zhao X. 2014. Display of Eimeria tenella EtMic2 protein on the surface of Saccharomyces cerevisiae as a potential oral vaccine against chicken coccidiosis. vaccine, 32, 1869-7186.

37. Tan L, Li Y, Yang X, Ke Q, Lei W, Mughal MN, Fang R, Zhou Y, Shen B, Zhao J. 2017. Genetic diversity and drug sensitivity studies on Eimeria tenella field isolates from Hubei Province of China. Parasite \& Vectors, 10(1), 137.

38. Tian XL, Tang XM, Qin M, Suo JX, Liu XY, Suo X. 2014. Analysis on the location of microneme 2 protein in sporozoites of Eimeria spp. by indirect immunofluorescence assay. Acta Parasitologica et Medica Entomologica Sinica, 21(4), 240-243 (in Chinese).

39. Tomley FM, Bumstead JM, Billington KJ, Dunn PP. 1996. Molecular cloning and characterization of a novel acidic microneme protein (Etmic-2) from the apicomplexan protozoan parasite Eimeria tenella. Molecular and Biochemical Parasitology, 79(2), 195-206.

40. Tomley FM. 1997. Techniques for isolation and characterization of apical organelles from Eimeria tenella sporozoites. Methods, 13, 171-176.

41. Wiersma HI, Galuska SE, Tomley FM, Sibley LD, Liberator PA, Donald RG. 2004. A role for coccidian cGMP-dependent protein kinase in motility and invasion. International Journal for Parasitology, 34(3), 369-380.

42. Williams RB. 2001. Quantification of the crowding effect during infections with the seven Eimeria species of the domesticated fowl: its importance for experimental designs and the production of oocyst stocks. International Journal for Parasitology, 31, 1056-1069. 
43. Wu SQ, Wang M, Liu Q, Zhu YJ, Suo X, Jiang JS. 2004. Construction of DNA vaccines and their induced protective immunity against experimental Eimeria tenella infection. Parasitology Research, 94, 332-336.

44. Xu Q, Song X, Xu L, Yan R, Shah MA, Li X. 2008. Vaccination of chickens with a chimeric DNA vaccine encoding Eimeria tenella TA4 and chicken IL-2 induces protective immunity against coccidiosis. Veterinary Parasitology, 156, 319-323.

45. Zhang J, Chen P, Sun H, Liu Q, Wang L, Wang T, Shi W, Li H, Xiao Y, Wang P, Wang F, Zhao X. 2014. Pichia pastoris expressed EtMic2 protein as a potential vaccine against chicken coccidiosis. Veterinary Parasitology, 205, 62-69.

46. Zhang L, Ma L, Liu R, Zhang Y, Zhang S, Hu C, Song M, Cai J, Wang M. 2012. Eimeria tenella heat shock protein 70 enhances protection of recombinant microneme protein MIC2 subunit antigen vaccination against $E$. tenella challenge. Veterinary Parasitology, 188(3-4), 239-246.

47. Zhang Z, Liu L, Huang J, Wang S, Lu M, Song X, Xu L, Yan R, Li X. 2016. The molecular characterization and immune protection of microneme 2 of Eimeria acervulina. Veterinary Parasitology, 215, 96-105.

Cite this article as: Yan M, Cui X, Zhao Q, Zhu S, Huang B, Wang L, Zhao H, Liu G, Li Z, Han H \& Dong H. 2018. Molecular characterization and protective efficacy of the microneme 2 protein from Eimeria tenella. Parasite 25, 60.

\section{O PARASTE}

An international open-access, peer-reviewed, online journal publishing high quality papers on all aspects of human and animal parasitology

Reviews, articles and short notes may be submitted. Fields include, but are not limited to: general, medical and veterinary parasitology; morphology, including ultrastructure; parasite systematics, including entomology, acarology, helminthology and protistology, and molecular analyses; molecular biology and biochemistry; immunology of parasitic diseases; host-parasite relationships; ecology and life history of parasites; epidemiology; therapeutics; new diagnostic tools.

All papers in Parasite are published in English. Manuscripts should have a broad interest and must not have been published or submitted elsewhere. No limit is imposed on the length of manuscripts.

Parasite (open-access) continues Parasite (print and online editions, 1994-2012) and Annales de Parasitologie Humaine et Comparée (1923-1993) and is the official journal of the Société Française de Parasitologie. 